\title{
Exploración del concepto naturaleza a partir de redes semánticas naturales en estudiantes de educación básica
}

\section{Nature concept exploration from natural semantic networks in elementary education students}

\author{
Blanca Silvia Fraijo-Sing ${ }^{* *}$ \\ ORCID: 0000-0001-6794-387X \\ Universidad de Sonora, México
}

\section{Laura Fernanda Barrera-Hernández ${ }^{* *}$}

ORCID: 0000-0002-1646-2037

Instituto Tecnológico de Sonora, México

Cesar Octavio Tapia-Fonllem ORCID: 0000-0002-5668-8540

Anaís Ortiz-Valdez

ORCID: 0000-0002-8775-036X

Universidad de Sonora, México

Recibido: 1 de agosto de 2017 Revisado: 11 de noviembre de 2017

Aceptado: 9 de enero de 2018

\section{Resumen}

El objetivo de la presente investigación fue describir la concepción de la palabra naturaleza desde la perspectiva de niños que estudian educación básica, así como analizar las similitudes y diferencias según el grado escolar. Participaron 272 alumnos de educación básica (172 mujeres y 94 hombres). Como resultado general, se obtuvo tamaño de red (TR) de 203 palabras definidoras de Naturaleza y se incluyeron en el núcleo de red 21 palabras. Aquellas con mayor peso semántico fueron: árboles, plantas, cuidar, sol, agua, flores, tierra, entre otras. Por otra parte, se analizaron redes por grado escolar, donde destacan las palabras que se presentan en todos los núcleos (conjunto SAM) de las redes semánticas: árboles, animales, agua y sol. Asimismo, se observó un aumento en el tamaño de las redes semánticas en

Artículo de investigación. DOI: https://doi.org/10.15332/s1794-9998.2018.0002.03

Correspondencia: Blanca Silvia Fraijo-Sing, Doctora en Ciencias Sociales, UAS. Profesor investigador del Departamento de psicología y ciencias de la comunicación, Universidad de Sonora. Sus líneas de investigación son educación, familia y medio ambiente. Miembro del Sistema Nacional de Investigadores (SNI), CONACYT - México. Correo electrónico. Blancafraijo@gmail.com; Dirección postal: Blvd. Reforma y Colosio, Edificio 7-F. Unidad Integral de Posgrado Cubículo 2, Hermosillo, Sonora, México.

Laura Fernanda Barrera-Hernández, Doctora en Ciencias Sociales, UNISON. Profesor investigador del Departamento de psicología., Instituto Tecnológico de Sonora. Correo electrónico: laura.barrera@itson.edu.mx Dirección postal: 5 de Febrero 818 sur, Col. Centro, Ciudad Obregón, Sonora, México. 
función del grado escolar, excluyendo la red de primero grado de primaria que incluía palabras no pertinentes al estímulo.

Palabras clave: Naturaleza, red semántica, psicología ambiental, niños.

\begin{abstract}
The goal of this study was to describe the conception of "nature" in 272 primary school children, and to analyze similarities and differences according to grade. (172 women and 94 men). As a general result, 203 was the network size of words defining "nature", and 21 words were included in the network core; those with greater semantic weight were: trees, plants, caring, sun, water, flowers, land, among others. On the other hand, networks were analyzed by grade, and words common to all nuclei (SAM set) of the semantic networks were trees, animals, water and sun. Also, an increase in the size of semantic networks was observed according to grade, excluding the network of the first grade of Elementary School that included words not pertinent to the stimulus.
\end{abstract}

Keywords: Nature, semantic network, environmental psychology, children.

\section{Introducción}

La investigación cualitativa busca la subjetividad, además de explicar y comprender las interacciones y los significados subjetivos individuales 0 grupales (Álvarez-Gayou, 2003). Las palabras, las oraciones y el discurso en cualquiera de sus formas conllevan significado (De Vega, 2002) y a su vez, el significado puede ser denotativo y connotativo. Es considerado denotativo cuando es asignado de manera formal y general, y por otra parte se considera connotativo cuando se desarrolla con referencia a un objeto, de manera subjetiva, particular y contextual, tal como el significado psicológico, que puede tener una función mediadora entre el objeto y las conductas (Moreno, 1999); esta investigación se centrará en el significado connotativo.

\section{Significado y significado psicológico}

Siendo uno de los aspectos fundamentales del lenguaje, el significado de los conceptos ha sido estudiado desde diversos puntos de vista. Así las posiciones teóricas y metodológicas varían desde las teorías de conductismo y mediación ortodoxos, hasta las interpretaciones cognitivas (Figueroa, González, \& Solís, 1976). Una forma de abordar el estudio de los significados es a través de las redes semánticas, donde, al considerar la riqueza de la red semántica generada y su relación con el proceso de reconstrucción en la memoria, se da el conocimiento de un concepto. Cuando se elabora una red semántica, entre más palabras adquiere la red el significado de un concepto se amplía, por otra parte, los conceptos, cuyas redes semánticas son muy pequeñas, son considerados como conceptos sin sentido (Figueroa et al., 1976).

Por otra parte, el significado psicológico es un tema popular entre los investigadores en prácticamente todos los campos de las ciencias sociales y del comportamiento. Éste se centra en la reacción subjetiva de una persona o grupo en particular, éste significado subjetivo o reacción de codificación implica además de los componentes cognitivo-referenciales, también componentes afectivos de evaluación y se diferencia del significado léxico (lexical meaning) debido a que éste define lo que es una palabra en particular, basándose en la convención establecida de un lenguaje en específico (Szalay \& Bryson, 1974).

\section{Redes semánticas}

Reyes (1993) define a la red semántica como el grupo de nociones seleccionadas por la memoria a través de un proceso reconstructivo que permi- 
te la evaluación subjetiva de temas o estímulos y que resulta en el significado psicológico. Esta técnica se basa en la premisa de que existe una organización interna de la información en la memoria, en forma de red donde las palabras forman relaciones que en conjunto otorgan el significado.

Suárez de Figueroa y Gómez (2008) señalan que las redes semánticas o redes conceptuales son una técnica basada en la forma de presentación del conocimiento basado en relaciones. Esta técnica, permite obtener información sobre las concepciones y percepciones que tienen las personas con relación al problema de estudio ( $\mathrm{Na}$ varro \& Garrido, 2006). Vera, Pimentel y Batista (2005) sugieren el uso de la técnica de redes semánticas cuando se desconoce o se tienen dudas sobre el significado de una comunidad acerca de un objeto, sea por su heterogeneidad, la búsqueda de generalización, o debido a que las observaciones previas revelan que es un concepto polémico.

La red semántica de un concepto está conformada por los conceptos organizados en la memoria; sin embargo, esta red no se debe exclusivamente a vínculos asociativos; sino que son dadas por un proceso organizador de la memoria, donde la selección de los elementos de la red no se basa en la fortaleza de la asociación, sino más bien, en la clase y las propiedades de tales elementos (Figueroa et al., 1976).

Es posible estudiar las redes semánticas a través de las redes artificiales, donde se presentan listas de palabras para definir un concepto a los participantes quienes deben elegir la que mejor lo define y valorizar cada una de ella para establecer jerarquizaciones, y por otra parte, están también las redes semánticas naturales que consisten en presentar conceptos sobre una palabra o tema estímulo, del cual se quiere indagar el significado, en esta modalidad en vez de presentar la lista de palabras la persona define el concepto y le otorga valor (Sánchez, De la Garza y López, 2011).

\section{Desarrollo cognitivo e investigaciones de redes semánticas en niños}

Los estudios sobre el pensamiento en la infancia se enfocan en la formación y categorización de conceptos, los conceptos son considerados categorías que agrupan objetos, eventos, y características con base en propiedades comunes. Se ha encontrado que los bebés forman conceptos desde las primeras etapas de su desarrollo, a los tres meses pueden hacer categorizaciones perceptuales. No obstante, los primeros conceptos de los bebés son amplios; durante los dos primeros años de vida estos conceptos amplios comienzan a ser diferenciados de forma gradual (Santrock, 2007).

Entre las investigaciones que estudian las estrategias semánticas en niños, se encuentra la desarrollada por Introzzi, Urquijo, Richard's, Canet-Juric y Richaud (2012) quienes concluyeron a partir de su investigación con 185 niños de entre 6 y 10 años, que en la medida que se incrementa el nivel de escolaridad, los niños tienden a implementar un número mayor de estrategias semánticas, tales como agrupación en categorías o establecimiento de relaciones semánticas entre los elementos a memorizar en tareas de aprendizaje y recuerdo. Por otra parte, revelan que no observaron diferencias entre los resultados de los niños de primer, segundo y tercer año, sino que el avance y la diferencia se presentan en los alumnos de cuarto año con relación a los tres años anteriores, etapa escolar en la que el uso de estas estrategias parece ser más sistemático y consistente.

Por otra parte Torres, Piñeiro, Morenza e Inguanzo (2000) realizaron un estudio de redes semánticas con niños de entre 3 y 6 años de edad donde se les presentaron seis términos estímulos: alimentos, animales, bebidas, frutas, herramientas, e instrumentos musicales; los resultados indican que las categorías alimentos (61) y animales (43) son las que más ejemplares mostraron y que este resultado, está dado por el alto nivel de generalidad e inclusividad que tienen ambas categorías, la categoría que presentó ejemplares es instrumentos musicales (16). Asimismo, indicaron que no se encontraron grandes diferencias en la cantidad de 
ejemplares que producen los niños de 3 y 4 años en cada categoría, en algunas de estas categorías, los niños de 4 años producen alrededor de tres ejemplares más que los niños de 3 años (salvo frutas y herramientas). Finalmente, los autores concluyen que antes de los cinco años, los niños son capaces de agrupar objetos en categorías, que contienen ejemplares que incluso los adultos incluyen, y en ocasiones, algunos elementos incluidos no son pertinentes.

\section{Investigaciones de redes semánticas de términos relacionados al medio ambiente}

Sánchez, De la Garza y López (2011) mencionan que existen investigaciones donde se aborda el tema del medio ambiente, sin embargo, se han centrado en aspectos específicos de educación ambiental o sobre su cuidado, por lo que existen carencias sobre la representación del tema del medio ambiente que permitan detectar las similitudes y diferencias entre grupos de contraste; por lo que estos autores recomiendan indagar acerca de la temática ambiental y utilizando la técnica de las redes semánticas naturales como una forma de evaluar los conocimientos que tienen los grupos.

Al respecto de los estudios de redes semánticas naturales acerca de la temática ambiental se encuentra la investigación realizada por Navarro y Garrido (2006) quienes desarrollaron un estudio con 200 estudiantes de secundaria en Veracruz (México) respecto a los conceptos: contaminación, ecología, reciclaje, reutilizar, medio ambiente, y basura. Observaron que el estímulo medio ambiente obtuvo el tamaño de red semántica más grande (valor J) de 195 palabras; mientras que ecología el más bajo, con 112 palabras que integran la red semántica. Asimismo, estos autores indicaron que al definir la palabra medio ambiente los alumnos la relacionaron principalmente con los conceptos de animales-fauna, flora, vegetación, naturaleza, agua, aire y gente.

Milfont (2010) desarrolló una investigación acerca de los términos "conservación del medio ambien- te" y "uso del medio ambiente" con 80 personas de 16 países diferentes que tenían una edad media de 33,68 años. Entre los resultados encontró un total de 217 definidoras para el estímulo "conservación del medio ambiente" y 195 para "uso del medio ambiente" por lo que concluyó que los participantes están más familiarizados con el concepto de conservación, que con el de uso o explotación de medio ambiente, las diez definidoras principales de conservación del medio ambiente fueron: conservación, reciclar, proteger, bosques, futuro, salvar, árboles, necesario, difícil y reservas, y por otra parte, en cuanto a las definidoras de mayor relevancia respecto al estímulo uso del medio ambiente estuvieron: explotación, sostenibilidad, uso, polución, agua, agricultura, bosques, precauciones, abuso y humanos. Finalmente, observó que la conservación del medio ambiente se asoció principalmente con definidoras positivas, mientras que el uso del medio ambiente se asoció con definidoras tanto positivos como negativos.

Sánchez, De la Garza y López (2011) investigaron con redes semánticas diversos conceptos relacionados al medio ambiente: ciudad, contaminación, deforestación, naturaleza, ecología, medio-ambiente y reciclaje, donde compararon las aportaciones de estudiantes de biología y psicología. En la red del estímulo Naturaleza derivada de las participaciones de los estudiantes de psicología tuvo un valor J (tamaño de la red) de 165 palabras donde las definidoras más importantes fueron: animales, árboles, plantas, verde, vida, ríos, bonita, fauna, bosques y flora; por otra parte respecto a las aportaciones de los estudiantes de biología el término naturaleza tuvo un valor $\mathrm{J}$ de 177 definidoras, donde las destacadas fueron: animales, plantas, vida, verde, bonita, flora, fauna, ambiente, agua y árboles. Al finalizar la investigación, los autores señalan diferencias que tipifican a los grupos de acuerdo con las experiencias académicas, es decir los estudiantes de biología tendieron a definir los conceptos con términos revisados en su ámbito de estudio y por otra parte los estudiantes de psicología realizaron su representación con palabras que tiene que ver con el conocimiento cotidiano del área ambiental. 


\section{Naturaleza}

Según el diccionario de lengua española (2016) la palabra naturaleza posee diversas definiciones: principio generador del desarrollo armónico y la plenitud de cada ser en cuanto tal ser, siguiendo su propia e independiente evolución; conjunto de todo lo que existe y que está determinado y armonizado en sus propias leyes, virtud, calidad o propiedad de las cosas, entre otras.

Por otra parte, el término Naturaleza según el diccionario de Oxford (2016) asume siete definiciones, aquí se describen las primeras tres: conjunto de las cosas que existen en el mundo o que se producen o modifican sin intervención del ser humano, principio creador y organizador de todo lo que existe y conjunto de los constituyentes geográficos de la Tierra (el campo, los bosques, las montañas, los ríos, las aguas, etc.

Kaplan y Kaplan (1989 citado en Aragonés Tapia \& Amérigo, 2010) se refieren al medio natural con el término más genérico aún de naturaleza, este término hace referencia al conjunto de elementos vivos e inertes, que conforman un hábitat formado por espacios y recursos donde es menos apreciable la actividad humana, en este sentido el medio natural forma parte de un continuo cuyo polo opuesto se encontraría en el medio construido, cuyo prototipo es el medio urbano.

El objetivo de la presente investigación consiste en describir la concepción de la palabra naturaleza desde la perspectiva de niños que estudian educación básica. Además, se analiza el tamaño de las redes semánticas y las principales definidoras según el grado escolar. Se espera que conforme aumenta el grado escolar de los niños, el tamaño de la red aumente.

\section{Método}

\section{Participantes}

Participaron 272 alumnos de educación básica de una institución educativa del sector público en la ciudad de Hermosillo, Sonora. De los cuales 172 fueron mujeres y 94 fueron hombres. Respecto al grado escolar 44 son de primero (18 hombres y 26 mujeres), 41 de segundo (12 hombres y 29 mujeres), 54 de tercero (14 hombres y 39 mujeres), 49 de cuarto (18 hombres y 31 mujeres), 34 de quinto (14 hombres y 19 mujeres), 47 de sexto (18 hombres y 28 mujeres), y 3 alumnos no especificaron; las edades de los participantes oscilaban entre 6 y 12 años $(M=8,70$ años, $D E=1,88)$.

\section{Procedimiento}

Para la recolección de los datos se utilizó la técnica de redes semánticas naturales donde se presentó como frase estímulo "Naturaleza" en un formato con espacio para escribir palabras descriptoras y para jerarquizarlas en función de su relevancia.

Una vez que se obtuvieron los permisos de acceso a los participantes y padres de familia, se acudió a los salones de clase de los niños y se realizó la invitación para participar en la investigación. Se les dio la indicación de escribir las palabras que asociaran al concepto de naturaleza, y se insistió en utilizar sólo una palabra para definir el concepto.

Después se solicitó que realizaran la valoración de cada palabra del 1 al 5 , siendo 1 la palabra más importante y 5 la menos importante. El tiempo para realizar la actividad fue de 5 minutos aproximadamente.

Posterior a esto se calculó el tamaño de la red, el peso semántico, el núcleo de la red y la distancia semántica cuantitativa.

Algunas de las palabras definidoras fueron combinadas en categorías integradoras debido a las relaciones de sinonimia entre ellas, tales como: "cuidar" y "proteger", "zacate", "pasto" y "césped", "plantas" y "flora", "animales" y "fauna", "ambiente" y "medio ambiente", así como "personas" y "seres humanos".

Adicionalmente, se creó un grupo de "animales" donde se integró a las palabras que referían a animales tales como: animales, zorrillo, zorro, tigres, ratones, ranas, perro, peces, pato, abejas, pája- 
ros, paloma, osos, monos, leones, insectos, gato, gansos, caballo, fauna, ardilla, aves, conejo.

\section{Resultados}

La participación del grupo total de niños generó un tamaño de red (TR) de 203 palabras definidoras de Naturaleza y al realizar la suma de la ponde- ración de la frecuencia por la jerarquización para obtener el peso semántico se incluyeron en el núcleo de red 21 palabras; la definidora 22 ya no se incluyó debido a que representa el punto de quiebre donde el peso semántico se vuelve asintótico. Las palabras que resultaron con un mayor peso semántico fueron: árboles, plantas, cuidar, sol, agua.

Tabla 1.

Peso y distancia semántica del conjunto de definidoras de Naturaleza en alumnos de primaria.

\begin{tabular}{|c|c|c|c|c|c|}
\hline \multicolumn{3}{|c|}{ Versión original } & \multicolumn{3}{|c|}{ Versión animales agrupados } \\
\hline Definidora & PS & DS & Definidora & PS & DS \\
\hline Árboles & 1371 & 0 & Animales & 1754 & 0 \\
\hline Agua & 300 & 78 & Árboles & 1371 & 22 \\
\hline Plantas & 190 & 86 & Plantas & 945 & 46 \\
\hline Mariposa & 148 & 89 & Agua & 820 & 53 \\
\hline Primavera & 113 & 92 & Flores & 640 & 64 \\
\hline Ríos & 110 & 92 & Cuidar & 541 & 69 \\
\hline Flores & 104 & 92 & Sol & 307 & 82 \\
\hline Animales & 102 & 93 & Zacate & 270 & 85 \\
\hline Sol & 63 & 95 & Aire & 259 & 85 \\
\hline Zacate & 49 & 96 & Mariposa & 172 & 90 \\
\hline Frutas & 46 & 97 & Tierra & 146 & 92 \\
\hline Verde & 42 & 97 & No Contaminar & 139 & 92 \\
\hline Tierra & 40 & 97 & Frutas & 130 & 93 \\
\hline Vida & 40 & 97 & Primavera & 113 & 94 \\
\hline Rosas & 39 & 97 & Ríos & 110 & 94 \\
\hline 3 r's & 38 & 97 & Naturaleza & 99 & 94 \\
\hline Reusar & 38 & 97 & Verde & 86 & 95 \\
\hline Cielo & 32 & 98 & Hojas & 75 & 96 \\
\hline Abeja & 31 & 98 & Reusar & 71 & 96 \\
\hline Peces & 30 & 98 & Oxígeno & 69 & 96 \\
\hline Valor $\mathrm{J}=203$ & & & Valor $\mathrm{J}=176$ & & \\
\hline
\end{tabular}

Fuente: elaboración propia. 
En los resultados de la red semántica de la palabra estímulo "naturaleza" por grupo, se encontró en el grupo de los niños de primer grado de primaria, un tamaño de red (TR o valor J) de 78 palabras definidoras y al realizar la suma de la ponderación de la frecuencia por la jerarquización para obtener el peso semántico se incluyeron en el núcleo de red 20 palabras; la definidora 21 ya no se incluyó debido a que representó el punto de quiebre donde el peso semántico se volvía asintótico.

En segundo de primaria, el tamaño de red (TR) fue de 51 palabras y el núcleo de red se conformó por 23 palabras. Para tercero de primaria, el tamaño de la red (TR) fue de 50 palabras y el núcleo de red fue de 20 palabras. En los resultados de la red semántica de los alumnos de cuarto de primaria, un tamaño de red (TR) de 58 palabras definidoras de Naturaleza y al realizar la suma de la ponderación de la frecuencia por la jerarquización para obtener el peso semántico se incluyeron en el núcleo de red 28 palabras; la definidora 9 ya no se incluyó debido a que representó el punto de quiebre donde el peso semántico se volvía asintótico. En los resultados de la red semántica de los alumnos de quinto de primaria, la red se integró por 57 palabras definidoras y se incluyeron en el núcleo de red de 25 palabras.

Finalmente, en los resultados de los alumnos de sexto de primaria, se generó un tamaño de red (TR) de 81 palabras y el núcleo de red fue integrado por 26 palabras.

Tabla 2.

Peso y distancia semántica del conjunto de definidoras de Naturaleza por grado.

\begin{tabular}{lcclccccc}
\hline \multicolumn{1}{c}{ Primer grado } & \multicolumn{1}{c}{ Segundo grado } & \multicolumn{3}{c}{ Tercer grado } \\
\hline \multicolumn{1}{c}{ Definidora } & PS & DS & \multicolumn{1}{c}{ Definidora } & PS & DS & Definidora & PS & DS \\
\hline Árboles & 158 & 0 & Árboles & 251 & 0 & Árboles & 407 & 0 \\
\hline Agua & 109 & 31 & Animales & 211 & 16 & Animales & 315 & 23 \\
\hline Flores & 108 & 32 & Plantas & 179 & 29 & Plantas & 213 & 48 \\
\hline Mariposas & 75 & 53 & Flores & 151 & 40 & Flores & 204 & 50 \\
\hline Animales & 67 & 58 & Sol & 71 & 72 & Agua & 174 & 57 \\
\hline Oso & 45 & 72 & Primavera & 62 & 75 & Sol & 144 & 65 \\
\hline Limpio & 44 & 72 & Mariposas & 54 & 78 & Zacate & 57 & 86 \\
\hline Zacate & 41 & 74 & Zacate & 46 & 82 & Ríos & 54 & 87 \\
\hline Plantas & 39 & 75 & Agua & 37 & 85 & Primavera & 51 & 87 \\
\hline Sol & 39 & 75 & Insectos & 37 & 85 & Aire & 46 & 89 \\
\hline Mamá & 37 & 77 & Verano & 32 & 87 & Mariposas & 42 & 90 \\
\hline Conejo & 28 & 82 & Rosas & 30 & 88 & Cuidar & 41 & 90 \\
\hline Hojas & 28 & 82 & Naturaleza & 27 & 89 & Frutas & 33 & 92 \\
\hline Aves & 24 & 85 & Hojas & 24 & 90 & Cielo & 25 & 94 \\
\hline Pájaros & 24 & 85 & Pájaros & 23 & 91 & Tierra & 23 & 94 \\
\hline Casa & 23 & 85 & Peces & 22 & 91 & Pájaros & 21 & 95 \\
\hline Luz & 23 & 85 & Frutas & 21 & 92 & No Contaminar & 20 & 95 \\
\hline Abejas & 22 & 86 & Tierra & 21 & 92 & Nubes & 20 & 95 \\
\hline & & & & & & & & \\
\hline
\end{tabular}




\begin{tabular}{|c|c|c|c|c|c|c|c|c|}
\hline \multicolumn{3}{|c|}{ Primer grado } & \multicolumn{3}{|c|}{ Segundo grado } & \multicolumn{3}{|c|}{ Tercer grado } \\
\hline Frutas & 19 & 88 & Luz & 17 & 93 & Abejas & 16 & 96 \\
\hline Niños & 19 & 88 & Basura & 16 & 94 & Hojas & 14 & 97 \\
\hline Valor $\mathrm{J}=78$ & & & Valor $\mathrm{J}=51$ & & & Valor $\mathrm{J}=50$ & & \\
\hline
\end{tabular}

\begin{tabular}{|c|c|c|c|c|c|c|c|c|}
\hline \multicolumn{3}{|c|}{ Cuarto grado } & \multicolumn{3}{|c|}{ Quinto grado } & \multicolumn{3}{|c|}{ Sexto grado } \\
\hline Definidora & PS & DS & Definidora & PS & DS & Definidora & PS & DS \\
\hline Árboles & 294 & 0 & Animales-Fauna & 226 & 0 & Agua & 409 & 0 \\
\hline Animales & 228 & 22 & Árboles & 195 & 14 & Animales & 209 & 49 \\
\hline Plantas & 196 & 33 & Plantas & 186 & 18 & Plantas & 115 & 72 \\
\hline Agua & 149 & 49 & Agua & 102 & 55 & Árboles & 103 & 75 \\
\hline Flores & 126 & 57 & Flores & 44 & 81 & Aire & 85 & 79 \\
\hline Aire & 98 & 67 & Oxígeno & 39 & 83 & Reusar & 71 & 83 \\
\hline Zacate & 84 & 71 & Frutas & 27 & 88 & Sol & 60 & 85 \\
\hline Tierra & 55 & 81 & Personas & 24 & 89 & No Tirar Basura & 53 & 87 \\
\hline Basura en su Lugar & 38 & 87 & Tierra & 22 & 90 & 3 R's & 38 & 91 \\
\hline Cuidar & 38 & 87 & Aire & 20 & 91 & Cuidar Ambiente & 36 & 91 \\
\hline Bonita & 33 & 89 & Mundo & 17 & 92 & Personas & 33 & 92 \\
\hline Cuidar Animales & 33 & 89 & Natural & 17 & 92 & Reciclar & 33 & 92 \\
\hline Cuidar Plantas & 32 & 89 & Vida & 17 & 92 & Cuidar/Proteger & 29 & 93 \\
\hline Oxígeno & 30 & 90 & Arbustos & 16 & 93 & Reducir & 28 & 93 \\
\hline Cuidar Agua & 29 & 90 & Naturaleza & 16 & 93 & Cuidar Árboles & 25 & 94 \\
\hline Personas & 23 & 92 & Recursos Naturales & 16 & 93 & Luz & 23 & 94 \\
\hline Reciclar & 21 & 93 & Zona Verde & 15 & 93 & Cuidar Animales & 19 & 95 \\
\hline Ambiente & 19 & 94 & Limpiar & 14 & 94 & Cuidarnos & 19 & 95 \\
\hline Cuidar Ambiente & 19 & 94 & Ríos & 14 & 94 & Ríos & 18 & 96 \\
\hline Mar & 19 & 94 & Seres Vivos & 14 & 94 & Tierra & 18 & 96 \\
\hline Valor $\mathrm{J}=58$ & & & Valor J = 57 & & & Valor $\mathrm{J}=81$ & & \\
\hline
\end{tabular}

PS = Peso semántico, DS = Distancia semántica, Valor $\mathrm{J}=$ Tamaño de la red

Fuente: elaboración propia.

Estos resultados indican que los niños asocian a la naturaleza principalmente con palabras que refieren a elementos de la naturaleza (árboles, plantas, animales, seres vivos, agua, mar, ríos, sol, vida, aire), adjetivos (verde, bonita), además del cuidado del medio ambiente y de algunos comportamientos pro-ecológicos (cuidar, proteger, no tirar basura, reciclar, reusar, etc.). 


\section{Conclusiones}

El objetivo de este estudio fue indagar el significado del término Naturaleza desde la perspectiva de niño, se encontró que las definidoras más relevantes y reiterativas son: árboles, animales, plantas, flores, cuidar, agua y bosque. Se esperaba que estas palabras se encontraran entre las definidoras de mayor peso semántico debido a que son elementos básicos que integran a la naturaleza, destaca el que incluyan el cuidado del ambiente, lo que pudiera hablar de un avance en la conciencia del cuidado del ambiente. Los resultados de esta investigación presentan coincidencias con los definidores de la red semántica de Naturaleza y de la red de Conservación de medio ambiente presentada por Milfont (2010) en los elementos: cuidar/proteger, bosques, árboles. Asimismo, se observan coincidencias en las definidoras de mayor peso semántico que forman el núcleo de red de esta investigación y las definidoras del estudio de Sánchez, De la Garza y López (2011) con estudiantes universitarios de psicología y biología, sin embargo difiere en el tamaño de la red o valor $J$ que es de 205 en la red semántica de niños, y de 165 en estudiantes de psicología y 177 en estudiantes de biología.

Por otra parte, los resultados respaldan también lo expuesto por Torres, Piñeiro, Morenza e Inguanzo (2000) quienes expresan que los niños menores de cinco años ya son capaces de agrupar objetos en categorías, y esas categorías contienen ejemplares que incluso los adultos incluyen, y que en ocasiones incluyen elementos no pertinentes, lo cual, también se presentó en esta investigación, específicamente, en la red semántica del grupo de los niños de primer grado de primaria, donde se observaron palabras no pertinentes al estímulo Naturaleza tales como: Disney, columpio, febrero, caca y película.

Respecto al tamaño de la redes semánticas (valor J) de la palabra naturaleza, se percibió un incremento por grados, de 51 palabras en la red semántica de segundo grado y de 50 palabras en tercero, a 58 en cuarto grado y 57 en quinto año, mientras que el tamaño de la red de sexto grado fue de 81 palabras, lo que concuerda con los hallazgos de Introzzi, Urquijo, Richard's, Canet-Juric y Richaud (2012) quienes expusieron que a medida de que se incrementa el nivel académico se tiende al aumento de establecimiento de relaciones semánticas.

Del análisis de las palabras definidoras que integran la red semántica natural acerca del estímulo naturaleza, destacan los siguientes hallazgos: las palabras que se presentan en todos los núcleos de las redes semánticas (conjunto SAM) por grado aluden a árboles, animales, agua y sol, mientras que flores y tierra, aparecen en los núcleos de las redes semánticas por grado en cinco de los seis grados. Por otro lado, en la red general se menciona a los elementos aire, agua y tierra, sólo faltó hacer mención al fuego para que se consideraran los cuatro elementos de la naturaleza. Asimismo, se observó un aumento en el tamaño de la red en función del grado escolar, excluyendo la red de primero de primaria que incluía palabras no pertinentes al estímulo; adicionalmente se encontró que las personas o seres humanos, se presentan las redes semánticas de los grupos de cuarto, quinto y sexto grado. No obstante estos términos, no aparecen en el núcleo de la red general, lo que podría implicar que no están percibiendo a las personas como integrantes importantes de la naturaleza, y el considerarse como un ente externo a la naturaleza podría implicar futuros comportamientos anti-ambientales.

Gudynas (1999) señala que son muy pocos los análisis sobre el concepto de Naturaleza, además plantea que la visión de la naturaleza, es parte de la visión acerca de la sociedad y del progreso, y que para ser modificadas, es necesario debatir y reflexionar desde ámbitos abiertos de discusión. Desde el ámbito educativo Navarro y Garrido (2006) indican que el proceso educativo debe estimular un cambio en la construcción de significados relacionados con el cuidado del ambiente, así como contar con la certeza de que los docentes, en primera instancia, poseen una clara conceptualización de los mismos para no generar confusión en los alumnos.

Esta investigación presentó las concepciones de la Naturaleza que poseen los niños que estudian educación primaria, y estas podrían ser resultado 
de los conocimientos adquiridos en los diversos ámbitos en los que se relacionan los niños, educativo, social y familiar. Por lo anterior, se sugiere continuar con el análisis del significado de la naturaleza, además de la forma en la cual el concepto ha evolucionado a través del tiempo y en caso de considerarse pertinente, incluir estudios de las concepciones de los profesores, o del contenido de las asignaturas que incluyen temas ambientales.

\section{Referencias}

Álvarez-Gayou, J. L. (2003). Cómo hacer investigación cualitativa. Fundamentos y metodología. México, DF: Paidós educador

Aragonés Tapia, J. I., \& Amérigo, M. (2010). Psicología ambiental. Madrid: Ediciones Pirámide.

De Vega, M. (2002). Del significado simbólico al significado corpóreo. Estudios de Psicología, 23 (2), 153-174.

Diccionario de la Lengua Española. (2016). Ambiente. En red recuperado a partir de: http:// dle.rae.es/?id=QHIB7B3

Figueroa, J. G., González, E. G., \& Solís, V. M. (1976). An approach to the problem of meaning: Semantic networks. Journal of Psycholinguistic Research, 5(2), 107-115.

Gudynas, E. (1999). Concepciones de la naturaleza y desarrollo en América Latina. Persona y Sociedad, 13(1), 101-125. Recuperado de: http:// www.ecologiasocial.com/publicacionesclaes/ GudynasConcepcionesNaturalezaPSC199.pdf

Introzzi, I., Urquijo, S., Richard's, M., Canet-Juric, L., \& Richaud, M. (2012). Función ejecutiva y uso de estrategias semánticas en niños. Revista Latinoamericana de Psicología, 44(3), 31-40.

Milfont, T. L. (2010). The psychological meaning of preservation and utilization attitudes: A study using the natural semantic network technique. Psyecology, 1(1), 123-136.
Moreno, A. (1999). El significado psicológico de conceptos relativos a la educación ambiental. Revista Interamericana de educación de Adultos, 1(1-3), 92-109.

Navarro, R. E., \& Garrido, M. D. S. R. (2006). Construyendo el significado del cuidado ambiental: un estudio de caso en educación secundaria. REICE: Revista Electrónica Iberoamericana sobre Calidad, Eficacia y Cambio en Educación, 4(1), 52-70.

Oxford dictionaries. (2016). Naturaleza. En red recuperado de: https://es.oxforddictionaries. com/definicion/naturaleza

Reyes, L. I. (1993). Las redes semánticas naturales, su conceptualización y su utilización en la construcción de instrumentos. Revista de psicología social y personalidad, IX(1), 81-97.

Sánchez, M., De la Garza, A., \& López, E. (2011). Redes semánticas naturales del tema de medio ambiente en dos grupos de contraste. $R e$ vista Mexicana de Investigación en Psicología, 3(1), 60-71.

Santrock, J. W. (2007). Desarrollo infantil. México: McGraw-Hill Interamericana

Suárez de Figueroa, M., \& Gómez, A. (2008). Redes semánticas y marcos. En Palma, J. \& Marín, R. Inteligencia Artificial: técnicas, métodos y aplicaciones. España: McGraw Hill

Szalay, L. B., \& Bryson, J. A. (1974). Psychological meaning: Comparative analyses and theoretical implications. Journal of personality and social psychology, 30(6), 860.

Torres, R., Piñeiro, A., Morenza, L., \& Inguanzo, G. (2000). Estudio de seis categorías semántica en niños pequeños. Revista cubana de psicología, 17(2), 106-113.

Vera, J., Pimentel, C., \& Batista, F. (2005). Redes semánticas: Aspectos teóricos, técnicos, metodológicos y analíticos. Ra Ximhai, 3(1), 439-451. 